\title{
Marketing digital, una herramienta para el emprendimiento de estudiantes universitarios
}

\author{
Fecha de recepción: 2020-06-22 • Fecha de aceptación:2020-07-27 • Fecha de publicación: 2020-10-10
}

Byron Arturo Loayza Cabrera ${ }^{1}$

Banco Pichincha, Ecuador bloayza@pichincha.com https://orcid.org/0000-0002-7284-6545

Carmen Olinda Paredes Lombeyda ${ }^{2}$

Tata Consultancy Services, Ecuador c.paredeslombeyda1@tcs.com https://orcid.org/0000-0002-2977-0522

Jorge Alberto Ortega Rodríguez ${ }^{3}$

CRM Consultoría, Ecuador

jalbortg@hotmail.com

https://orcid.org/0000-0002-5722-1723

Nicol Madelaine Lozano Gómez ${ }^{4}$

Grupo Mavesa, Ecuador

nlozano@mavesaec.com.ec

https://orcid.org/0000-0001-8314-6635

\section{RESUMEN:}

La actual situación a nivel mundial y la competitividad en el mercado ha puesto en manifiesto la necesidad de emprender y activar diferentes tipos de estrategias de marketing digital. La presente investigación muestra un análisis de la utilización de herramientas digitales por emprendedores, específicamente en estudiantes del noveno semestre de la Universidad Israel, siendo esta etapa universitaria decisiva para quienes desean desarrollar su negocio, basado en las técnicas aplicadas en el aula de clases. La investigación es de tipo descriptiva, se aplicó una encuesta a 61 emprendedores 
que posteriormente fue analizada mediante el método estadístico. En los resultados se evidencia que un mayor porcentaje de los encuestados tiene conocimiento en el marketing digital y que el uso de estas estrategias ha beneficiado su emprendimiento e incrementado sus ventas. A su vez, se reitera que las redes sociales siguen siendo el medio digital principal para publicitar.

El adecuado conocimiento y aplicación de estas estrategias y tecnologías permite alcanzar una mejor fidelización y conexión con los clientes potenciales.

\section{PALABRAS CLAVE: emprendimiento, marketing, medio digital, tecnología, ventas.}

\section{ABSTRACT:}

The current situation worldwide and the competitiveness in the market has highlighted the need to undertake and activate different types of digital marketing strategies. This research shows an analysis of the use of digital tools by entrepreneurs, specifically in students of the ninth semester of the Israel University, this university stage being decisive for those who wish to develop their business, based on the techniques applied in the classroom. The research is descriptive, a survey was applied to 61 entrepreneurs that was analyzed using the statistical method. The results show that a higher percentage of those surveyed have knowledge of digital marketing and that the use of these strategies has benefited their entrepreneurship, increasing their sales. In turn, it is reiterated that social networks continue to be the main digital medium for advertising.

Proper knowledge and application of these strategies and technologies allows for better loyalty and connection with potential customers. 


\section{Introducción}

En Latinoamérica, como lo cita Guillén (2018), países como Brasil y México son pioneros en la inversión de marketing digital, en el caso de Brasil, las empresas presentan más inversión con un $65,9 \%$ de usuarios online y un $16 \%$ de crecimiento en cuanto al uso de redes sociales, en comparación al año anterior. A su vez, México también tuvo un incremento del $65,3 \%$ en usuarios online y un $22 \%$ del uso de redes, a diferencia de años anteriores.

En el caso de Ecuador, se puede observar la gran atracción que genera el marketing digital en la mayoría de las medianas y grandes empresas, estas desarrollan cada vez más y mejores estrategias para publicitar de manera digital mediante grandes inversiones, con el objetivo de captar y mantener a sus clientes.

Salas, Acosta y Jiménez (2018) realizan un estudio sobre la importancia de la web 2.0 y la aplicación del marketing de atracción en las pequeñas y medianas empresas del Ecuador, reuniendo información de gran utilidad a través de la metodología de análisis científico, en donde concluyen que "en gran medida el éxito de muchas empresas se debe a la adopción de estas nuevas tendencias en su planificación y desarrollo estratégico" (p.78). Ellos sostienen que gran parte del éxito que obtienen los negocios de la ciudad están muy relacionados con la innovación y la correcta implementación de canales de marketing digital, lo que significa la automatización de los procesos claves, optimizando costos, tiempo y recursos en el manejo de sus ventas.

En el contexto temporal, el marketing digital nace entre 1990 y 1994 junto a las tecnologías de la información, lo compone un conjunto de estrategias aplicadas a la comercialización de productos o servicios, realizadas a través de medios digitales. Hoy en día, gracias a estas estrategias, los consumidores tienen un abanico de opciones al momento de decidirse por algún producto, pueden comparar varias ofertas de lo que requieren para suplir sus necesidades, por ende, es el cliente quien fija o precisa el valor del producto, pues las empresas se ven obligadas a segmentar el mercado y desarrollar servicios superiores para cada objetivo, es la era del marketing orientada al consumidor (Kotler y Armstrong, 2003).

En base a lo anterior, se puede decir entonces que este constituye una estrategia empresarial para atraer clientes, informar o dar a conocer sus ofertas al público en general, en este sentido, es necesario definir que la estrategia son acciones que se toman para vencer obstáculos y alcanzar los objetivos deseados, de esta manera, Roncancio (2019) define estrategia como "el puente que hay entre las políticas u objetivos más altos y las tácticas o acciones concretas para llegar a la meta".

Es preciso entonces destacar el origen etimológico de esta palabra, nace de los términos griegos "stratos" (ejército) "agein" (guía), lo que significa guía del ejército, en síntesis, la estrategia proviene de una concepción puramente militar, este término ha sido generalizando y con el paso del tiempo trasladado a otros campos del conocimiento como la administración empresarial y en los negocios (Roncancio, 2019).

En la presente investigación el emprendedor es parte fundamental. Según Martins (2020), en 
cita de Jolijt Tamanaha, Directora de Marketing y Finanzas en Fresh Prints: "los emprendedores se abren paso a través de una lista interminable de problemas con agallas, pasión y energía. Si bien es muy intenso, ser emprendedor significa que puedes vivir la vida aprendiendo una cantidad increíble de cosas y maximizas tu impacto en el mundo porque tienes que abordar los problemas más difíciles".

De igual modo, el término "emprendimiento" hace referencia a cambiar o innovar productos o servicios, de igual manera aspectos internos o externos de cada persona.

Impact Hub Madrid (2020) cita en su sitio web a la Comisión Europea que se refiere al emprendimiento como "la propensión a inducir cambios en uno mismo, la capacidad de aceptar y apoyar la innovación provocada por factores externo, de dar la bienvenida al cambio, de asumir la responsabilidad por las propias acciones (sean positivas o negativas), de terminar lo que se empieza, de saber en qué dirección se está yendo, de establecer objetivos y cumplirlos y de tener la motivación necesaria para el éxito".

Es importante establecer en base a lo descrito la importancia que debe dar el emprendedor al contenido que mostrará a los clientes sobre sus productos. Se debe tener en cuenta que el consumidor muestra interés o atención de manera limitada o corta por lo cual su marca tiene 8 segundos para atraer al cliente llamando su atención e incentivarlo a la compra mediante varios medios como anuncios en televisión, la radio, el correo electrónico, utilizando mensajes instantáneos y de textos (Young, 2017). Resulta imprescindible que las empresas planteen estrategias de Marketing de Contenidos.

A continuación, se resumen los pasos para establecer una estrategia de marketing de contenidos óptima (Figura 1). Se debe lograr de manera eficiente que los usuarios se identifiquen con el contenido que muestra la marca, el cual deberá dar respuestas y soluciones a todos sus problemas (Rasche, 2018).

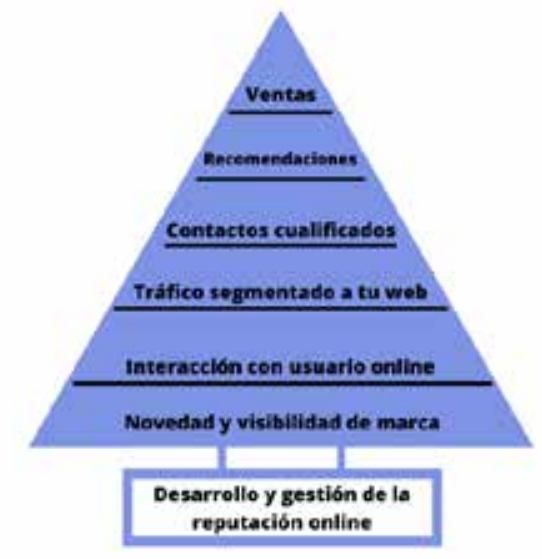

Figura 1. Pirámide del marketing de contenidos.

Fuente: elaboración propia 


\section{Principales plataformas y estrategias para el marketing digital}

\section{- Redes Sociales}

Las redes sociales son estructuras virtuales desarrolladas por personas $u$ organizaciones que crean un espacio de conexión en base a intereses, atractivos o valores en común. En estos medios de comunicación se enlazan relaciones entre personas, negocios y empresarios de una forma rápida, sin jerarquía o límites o barreras físicas. Estas plataformas virtuales del siglo XXI traen consigo una nueva forma para relacionarse entre empresarios y sus clientes, son de gran utilidad para generar contenido de marketing y publicitar gran variedad de productos y servicios, de manera que se pueda mantener una interesante interacción con potenciales clientes (RD Station, 2019).

\section{- Pauta publicitaria}

Se refiere a utilizar todos los recursos digitales disponibles para incrementar la presencia de marca en un público objetivo. Una pauta es un anuncio de cualquier índole que se promociona en un medio de comunicación, en este caso puede ser en las redes sociales, esta técnica de marketing forma parte de una campaña que incorpora varios medios digitales, además que realiza segmentación del mercado, presupuesto, los objetivos del anuncio, así también los tiempos estimados de la pauta publicitaria (Netbangers, 2019).

\section{- WhatsApp Business}

Esta aplicación gratuita para Android y Apple permite comunicarse de forma instantánea entre empresarios o negociantes y clientes en tiempo real (Ayuste, 2019).

En 2017 se hizo su lanzamiento con el objetivo de liberar a WhatsApp de mensajes spam por parte de negocios. Esta App permite a los empresarios mejorar la comunicación por la razón que aporta con el proceso rápido de trasmitir información al cliente, así mismo, este medio de comunicación digital se puede aplicar en la comunicación interna de las organizaciones. WhatsApp se ha encargado de facilitar procesos de comunicación, que anteriormente, en ocasiones eran impedimento para cerrar ventas (Ayuste, 2019).

\section{- Landing Page}

Se traduce a una página de llegada o de aterrizaje, es una página web que está diseñada y orientada para transmutar al visitante en un cliente potencial. Se llega a esta a través de un enlace, tiene como finalidad atraer visitas y convertirlos en potenciales clientes, para que posteriormente se produzca la venta deseada (NeoAttack, 2020).

\section{- Página web}

La bloguera Yuliana (2016) explica en su sitio web que a este se le conoce como un documento digital de carácter multimediático "es decir, capaz de incluir audio, video, texto y sus 
combinaciones (...) adaptado a los estándares de la World Wide Web (WWW) y a la que se puede acceder a través de un navegador Web y una conexión activa a Internet. Se trata del formato básico de contenidos en la red".

\section{- Píxel de Facebook}

En cuanto a esta herramienta de medición, Ivars (2017) sostiene que "es un fragmento de código javascript que insertamos en nuestra página web con el fin de realizar el seguimiento de las conversiones". Con la utilización del píxel podremos medir diversos aspectos tales como visitas en nuestra página web, artículos agregados al carrito de compra, artículos agregados a la lista de deseos, pago iniciado, compra de producto contenido visualizado, entre otros. Al momento de hacer su aplicación, el emprendedor deberá elegir lo que requiere lograr con esta estrategia de marketing.

\section{- Lead}

Un lead es un usuario que se encuentra registrado en una base de datos de cualquier organización, bajo su autorización y que previamente otorgó información de su persona por algún tipo de producto o servicio (Bel, 2020).

\section{- Shoppable post}

Según Cardona (2019) este "permite que las marcas etiqueten sus productos en fotos y vídeos y los usuarios los compren directamente en la propia red".

\section{Empresas exitosas en redes sociales}

Existe una gran cantidad de casos de éxito en empresas que han aprovechado al máximo los recursos tecnológicos, y que han tenido un indudable crecimiento en sus ventas, hoy son un modelo a seguir a nivel mundial, a continuación, se hace referencia a dos de ellas:

\section{- Deliveroo}

Es una compañía británica fundada en 2013 por Greg Orlowski y Will Shu que ofrece un servicio de comida a domicilio. Está presente en más de diez países. "Su potencial y creación se ha desarrollado en Internet, desde donde gestiona una parte importante de su trabajo. Cuenta con 800 empleados hasta la fecha" (Postedin, 2018).

Esta compañía tipo start-up "ha sabido sacar un tremendo partido a las telecomunicaciones y, en especial, a Internet, desde donde lanzó su campaña. Hoy es conocida en todo el mundo gracias a las redes sociales y al uso de anuncios en Youtube" (Postedin, 2018).

\section{- Uber}

A pesar que esta compañía ha generado polémica, la cobertura de Uber ha crecido en varios 
países gracias a su estrategia pionera en creación de una app que pone en contacto a personas que necesitan movilizarse y un conductor, por medio de Internet y el teléfono celular. La firma comenzó como un suplente al servicio convencional de taxis debido a las altas tasas que imponían en sus servicios, es así como luego de pocos años ha implementado el servicio de reparto o entrega de comida rápida a domicilio. Su nombre completo es Uber Technologies Inc. y fue creada en marzo del 2009 (Postedin, 2018).

Una vez mencionados estos dos casos de éxito, podemos establecer que el presente artículo corresponde al análisis de la importancia del uso de marketing digital aplicado en los negocios de los emprendedores, específicamente de los que forman parte de la comunidad de estudiantes del noveno semestre de las diferentes carreras de la Universidad Israel.

Esta muestra seleccionada se hace relevante ya que en la vida de los universitarios muchos deciden emprender con algún producto, aplicando los conocimientos adquiridos en el aula. En este sentido pueden realizarlo para tener independencia financiera y laboral.

Por esta razón se lleva a cabo el presente estudio, con el fin de precisar la utilidad que tienen las herramientas de marketing digital, considerando que las mismas constituyen un conjunto de técnicas aplicables en esta era digital, la cual ha cambiado por completo los patrones de venta, consumo y preferencias en los consumidores a nivel mundial.

\section{Metodología}

La investigación realizada tiene un alcance descriptivo. Tiene un enfoque cuantitativo y es un estudio de campo, ya que la principal fuente de recolección de datos es la encuesta. El instrumento tiene como objetivo conocer la percepción de los estudiantes en cuanto al conocimientos de marketing digital y su incidencia en las ventas, además de determinar cuál es el medio más utilizado por los emprendedores. La encuesta consta de 10 preguntas con opciones de selección múltiple analizadas mediante el método estadístico, estas fueron supervisadas por el director de Comunicación de la UISRAEL, Mg. Sebastián Pérez. La muestra seleccionada se conformó por estudiantes del noveno semestre, de un total de 177 estudiantes se identificó a 61 emprendedores que constituyeron la población grupo objetivo para el presente caso, exactamente 38 hombres y 23 mujeres.

Adicionalmente, se tomó en cuenta casos de éxito alcanzado por un emprendedor de la Universidad Israel, pretendiendo dar apoyo al crecimiento de los negocios que poseen menos experiencia o iniciativa de acción empresarial.

\section{Resultados}

En lo que se refiere al conocimiento sobre el marketing digital (Figura 2), el 62.3\% (38 estudiantes) del grupo objetivo manifiestan que si saben acerca de marketing digital en tanto que el $37.7 \%$ (23 estudiantes) manifiestan no conocer sobre esta herramienta, lo que podría estancar sus posibilidades de ser emprendimientos sostenibles en la actualidad que nos rodea. Se deberá 
tomar en cuenta el factor de inversión económica; donde se denota la importancia y diferencia del marketing orgánico y el marketing pagado, el primero consiste en la unión de estrategias metodológicas que facilitan la entrada, comprensión y crecimiento de la marca, este requiere de inversión de tiempo y compromiso, mas no se paga por este en los canales digitales, dicho mejor, aquí el cliente busca el producto mediante las diferentes plataformas, redes sociales o motores de búsqueda (Chili, 2019).

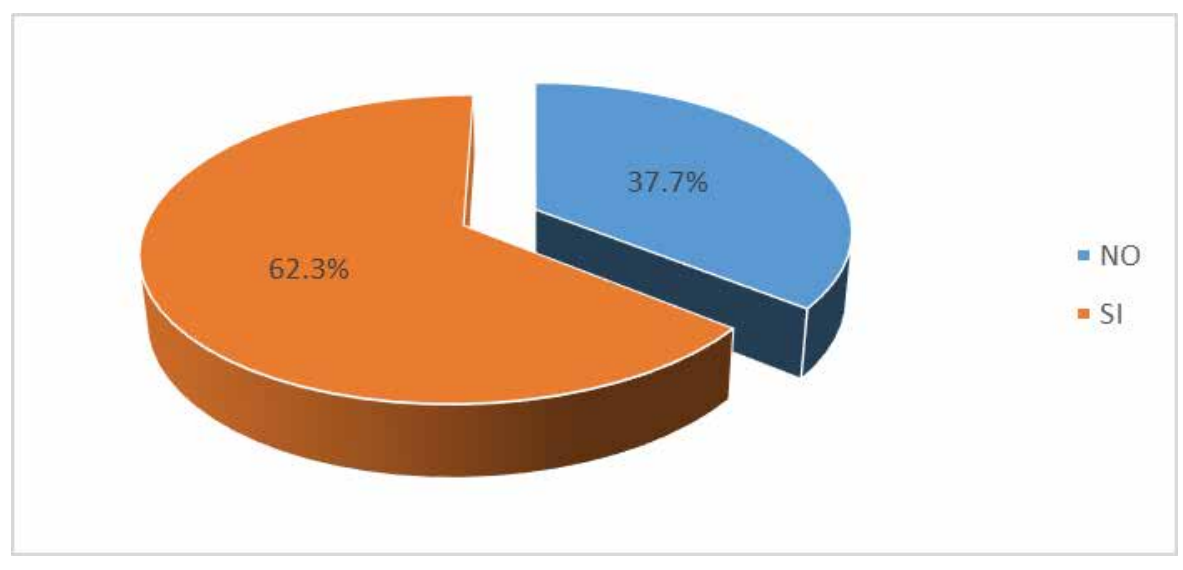

Figura 2. Emprendedores que conocen marketing digital

Fuente: elaboración propia

En la Figura 3 se evidencia que las redes sociales son el medio más utilizado por los emprendedores de la Universidad Israel con un $72.7 \%$. Estas resultan atractivas para publicar anuncios sobre sus bienes y servicios. Para las empresas con mayor autonomía económica existe la publicidad pagada, esta se realiza por medios especializados como Facebook Ads, Twitter ads, linkedln y por medio de Google Ads, llamado SEM (Search Engine Marketing) entre otros, en este tipo de marketing las plataformas trabajan mediante bases de datos o leads donde la marca se encarga de ofertar su producto basado en las posibles preferencias o necesidades del cliente y en las cuales se cobra por clic o ingreso (Chili, 2019).

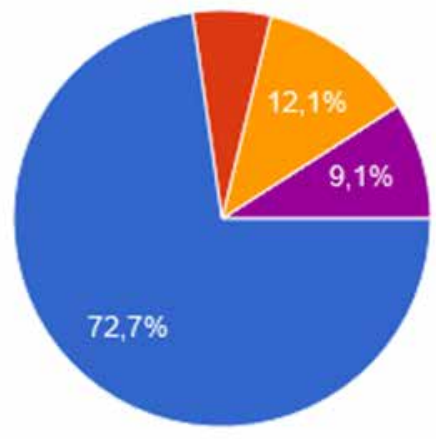

Redes sociales (Facebook, Instagram, Twitter, WhatsApp)

Páginas web

Boca a boca

Telemercadeo

Todas las anteriores

Figura 3. Medios digitales más utilizados en los emprendedores

Fuente: elaboración propia 
En cuanto al crecimiento obtenido gracias al uso del marketing (Figura 4), un $57.6 \%$ de los encuestados indica que han logrado mayor efectividad en sus ventas, en tanto que el $21.2 \%$ manifiesta que no, pues lo hacen de manera presencial sin utilizar canales digitales. Dicho en otras palabras, marketing digital es la publicidad de la nueva era, esta se adaptará, creará y mejorará continuamente con el fin de satisfacer el alto nivel de consumo que tienen los seres humanos, hoy en día la gran mayoría de comerciantes que van desde pequeños negocios caseros, hasta las grandes empresas, tienen conocimiento de esto (Guerrero, 2014).

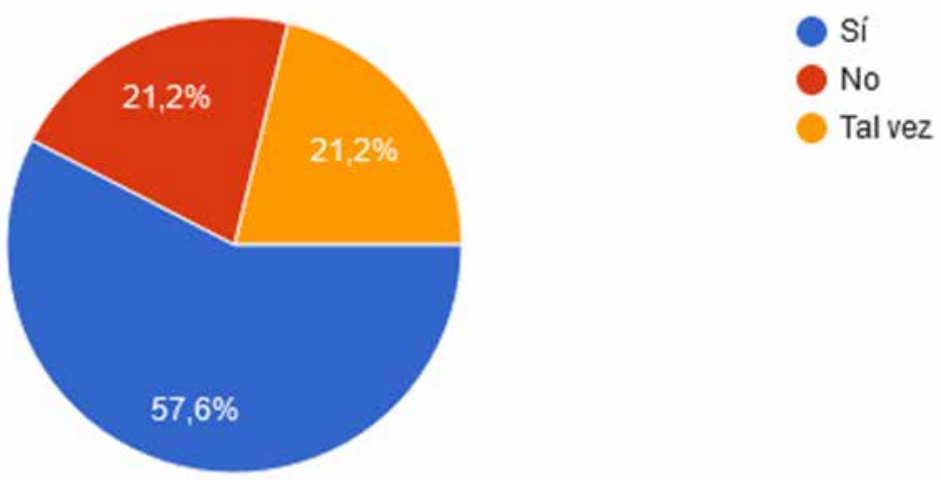

Figura 3. Crecimiento en ventas mediante el uso de Marketing digital

Fuente: elaboración propia

Un emprendedor o microempresario que utilice el Internet como medio al servicio del marketing digital podría alcanzar o expandir sus potenciales clientes y de la mano medir, mejorar y dar un seguimiento a las diferentes sugerencias o necesidades del mercado.

En base al testimonio de Roberto Olmedo, emprendedor del 9no semestre de la Universidad Israel y uno de los creadores del emprendimiento armariomóvil.com, se ratifica que el uso de marketing digital en la actualidad constituye una de las herramientas clave para el éxito de los emprendedores.

Gracias a la aplicación de las estrategias de marketing este emprendimiento acoge en su plataforma 319 tiendas afiliadas, más de 1234 productos disponibles para la venta, de marcas top nacionales e internacionales, como, por ejemplo, Lee, Pinto, Tennis, entre otras, además registra más de 407 clientes satisfechos, este negocio, desde sus inicios ha logrado un crecimiento en ventas del $200 \%$.

Considerando que la cultura ecuatoriana no había adquirido el hábito de comprar en línea, a diferencia de los países de Europa o Norteamérica, hoy día, y podría considerarse que por las medidas restrictivas en consecuencia de la pandemia mundial del COVID-19, este método de compras ha mejorado su aceptación de forma exponencial y está ganando mercado en el mundo de e-commerce. El marketing ha trasmutado a la era digital, en un mundo cada vez más movido por los canales tecnológicos, nacen nuevas formas de vender, publicitar y ofertar los productos y servicios (López, 2018). 


\section{Conclusiones}

La actual situación a nivel mundial y la competitividad en el mercado ha puesto en manifiesto la necesidad de emprender y activar diferentes tipos de estrategias de marketing digital, con el fin de mejorar su efectividad y crear un valor agregado intangible para el potencial cliente, esto con la intención crear una marca que pretende diferenciarse de la competencia. El marketing digital según los resultados ayuda a mejorar la eficiencia en ventas y visibilidad del negocio con herramientas efectivas que permiten perfilar a potenciales clientes por lo cual es imprescindible el conocimiento y uso de diferentes medios.

Para alcanzar buenos resultados se requiere el involucramiento de todas las herramientas que este brinda, sumado a la innovación y diferenciadores o valor agregado que los diferentes emprendimientos puedan otorgar al cliente final.

Como conclusión, podemos destacar en base a los resultados que las redes sociales aún se mantienen como la herramienta más utilizada del marketing digital, también se maneja la creación de página web o tienda virtual en Facebook, práctica e interactiva en donde el cliente pueda conocer los productos o servicios del emprendedor.

Si bien el estudio realizado se focalizó en los estudiantes emprendedores del 9no semestre de la UISRAEL, también se puede extrapolar y servirá de guía a otros emprendedores, además que permitirá tener el alcance información que necesitan sobre el marketing digital, su importancia, aplicación y casos de éxito. 


\section{Referencias}

Ayuste, M. (2019). ¿Qué es Whatsapp Business y por qué tu empresa debería utilizarla? https://bit. Iy/2E3HAMZ

Bel, O. (2020). ¿Qué es un lead, qué tipos hay y para qué sirven? Inboundcycle. https://www.inboundcycle.com/blog-de-inbound-marketing/que-es-un-lead

Cardona, L. (2019). Las 25 tendencias de marketing digital para 2020 (+ebook gratis). Ciberclick. https://www.cyberclick.es/numerical-blog/las-tendencias-de-marketing-digital

Chili. (2019). La diferencia entre el marketing orgánico y el marketing pagado. https://chili.pa/es/resources/marketing-digital/diferencia-entre-marketing-organico-marketing-pagado/

Guerrero, M. B. (2014). Marketing digital su aplicación y éxito (Bachelor's thesis, Quito, 2014.). http:// repositorio.usfq.edu.ec/handle/23000/4354

Impact Hub Madrid. (2020). Las 9 características de las personas emprendedoras. https://madrid. impacthub.net/2020/02/18/caracteristicas-de-las-personas-emprendedoras/\#: :text=La\%20Comisi\%C3\%B3n\%20Europea\%20defini\%C3\%B3\%20la,0\%20negativas)\%2C\%20de\%20terminar\%20lo

Ivars, A. (2017). ¿Qué es el Píxel de Facebook? IEBS. https://www.iebschool.com/blog/pixel-de-facebook-redes-sociales/

Kotler, P. y Armstrong, G. (2003). Fundamentos de Marketing. (6 $6^{\mathrm{a}}$ ed) México: Prentice Hall.

López, L. J. (2018). Propuesta de un plan de marketing apoyado en herramientas digitales en la web para empresa Pyme para promoción y venta de servicios médicos odontológicos en Clínicas Vega-Beltrán. UDLA, Facultad de posgrados. http://dspace.udla.edu.ec/bitstream/33000/9322/1/ UDLA-EC-TMGSTI-2018-02.pdf

Martins, N. (2020). ¿Qué es el emprendimiento? Definición y significado de emprendedor y emprendimiento. Oberlo. https://www.oberlo.es/blog/definicion-y-significado-emprendimiento

NeoAttack. (2020). Landing Page. https://neoattack.com/neowiki/landing-page/

Netbangers. (2019). Formatos de pauta en la estrategia de marketing digital de mi negocio. https:// www.netbangers.com/blog/formatos-de-pauta-en-la-estrategia-de-marketing-digital-de-mi-negocio 
Postedin. (2018). 5 marcas que destacan en su estrategia de marketing digital. https://www.postedin. com/blog/5-marcas-que-destacan-en-marketing-digital/

Rasche, E. (2018). Año nuevo...Web nueva. O cómo enfocar tu web para la captación de "leads". Marketing news.

RD Station. (2017). Redes Sociales. https://www.rdstation.com/mx/redes-sociales/

Roncancio, G. (2019). Estrategia: ¿Qué es? y las herramientas para crearla. Pensemos. https://gestion.pensemos.com/estrategia-que-es-y-las-herramientas-para-crearla

Salas, I. C., Acosta, m. M., \& Jiménez, m. E. (2018). Importancia del Marketing de Atracción 2.0, en las Pequeñas y Medianas Empresas de la ciudad de Guayaquil (Ecuador). Revista Espacios, 39(18).

Young, K. (2017). Marketing's New Mission: Engage and Entertain. American Bankers Association. ABA Banking Journal; 109 (5), 56-58.

Yuliana. (2016). Página web. Blogger. http://yulianabelen2001.blogspot.com/ 